\title{
AN INVERSE PROBLEM FOR THE TELEGRAPH EQUATION
}

\author{
A.B. Kurzhanski* \\ Moscow State University, Russia \\ kurzhans@mail.ru \\ M.M. Sorokina \\ Moscow State University, Russia \\ masha_sorokina2@mail.ru
}

\begin{abstract}
This paper deals with the problem of state estimation for a hyperbolic equation in the presence of unknown, but bounded disturbances, on the basis of information from sensors with finite-dimensional outputs. The object of investigation is the hyperbolic telegraph equation with energy dissipation. Observability properties similar to those introduced earlier for parabolic systems ([8]) are checked for various types of measurement sensors. Further on recurrent guaranteed minmax filtering procedures are introduced which give dynamic estimates of the current state of the system and dual control problems are indicated as well.
\end{abstract}

Keywords: Minmax filtering, telegraph equation, information set, sensors, observability.

\section{Introduction}

In this paper we consider the problem of state estimation for a system described by a hyperbolic equation of the "telegraph" type, with energy dissipation. This is to be done through available sensor measurements in the presence of unknown, but bounded disturbances.

We start with the the problem of observability which is the inverse problem of finding the final state for this system through available observations in the absence of any information on initial and boundary

*Funding provided by A.M. Liapunov French-Russian Institute, Project 02-01 and Russian Foundation for Basic Research (RFBR) grant N 03-01-00663. 
conditions and in the absence of disturbances. This problem may turn to be solvable depending on the paricular type of sensor applied. Conditions for observability are therefore investigated.

We further derive a filtering equation which gives a set-membership estimate of the state of the system under unknown disturbances subjected to a given quadratic bound. These equations also produce a vectorvalued estimate with respective bounds on the estimation error.

Finally some dual controllability problems are indicated.

\section{The Telegraph Equation and the Estimation Problem}

The telegraph equation is a PDE which describes, for example, an electric current transmission in the presence of wave aberration and depletion, namely ([2])

$$
\begin{aligned}
& v_{x x}=l c v_{t t}+(l g+r c) v_{t}+r g v \\
& i_{x x}=l c i_{t t}+(l g+r c) i_{t}+r g i
\end{aligned}
$$

where $i$ is the current intensity, $v$ is the voltage, $r$ is the resistance, $l$ is the induction, $c$ is the capacity and $g$ the conductivity. For $r, g=0$ it turns into a wave equation.

We further consider the following system:

$$
\begin{array}{ll}
u_{x x}=\frac{1}{v_{0}^{2}} u_{t t}-\left(\frac{\sigma_{1}+\sigma_{2}}{v_{0}^{2}}\right) \frac{\partial u}{\partial t}+\frac{\sigma_{1} \sigma_{2}}{v_{0}^{2}} u+f & \text { in } Q_{T} \\
\left.u\right|_{t=0}=\Phi(x) & \text { in } \Omega \\
\left.u_{t}\right|_{t=0}=\Psi(x) & \text { in } \Omega \\
\left.u\right|_{x=0}=\left.\mu_{1}(t) \quad u\right|_{x=l}=\mu_{2}(t) & t \in[0, T], x \in[0, l]=\Omega .
\end{array}
$$

Here $Q_{T}=\Omega \times(0, T), S_{T}=\partial \Omega \times(0, T)$ and $f$ is either a control or a disturbance (given or unknown).

An observation of the system performance is available through measurement sensors taken to be of the following types.

\section{Examples of sensors}

1 Spatially averaged $y(t)=\int_{D} g(\mathbf{x}) u(t, \mathbf{x}) d \mathbf{x}+\xi(t)$.

2 Pointwise $y(t)=u\left(t, \mathbf{x}^{0}\right)+\xi(t) \quad t \in\left[t_{1}, t_{2}\right] t_{1}>0$.

3 Dynamic pointwise $y(t)=u(t, \mathbf{x}(t))+\xi(t) \quad t \in\left[t_{1}, t_{2}\right] t_{1}>0$.

4 Distributed observation $y(\mathbf{x})=u(\theta, \mathbf{x})+\xi(\mathbf{x})$.

5 Dynamic spatially averaged $y(t)=\int_{O_{\varepsilon}(\mathbf{x}(t))} g(t, \mathbf{x}) u(t, \mathbf{x}) d \mathbf{x}+\xi(t)$. 
Then the measurement equation may be presented as

or

$$
\begin{gathered}
y(t)=G_{1}(t) u(\cdot, t)+\xi(t), \quad t_{0} \leq t \leq T \\
y(x)=G_{2} u(x, \cdot)+\xi(x), \quad 0 \leq x \leq l,
\end{gathered}
$$

where $G_{1}(t)\left(u(\cdot, t) \rightarrow \mathbf{R}^{m}\right), G_{2}\left(u(\cdot, \cdot) \rightarrow\left\{L_{2}^{m}(0, l), H_{1}(0, l)\right\}\right)$ are the measurement maps given by one of the sensors of the above or a combination of these. The disturbance in equations $(4,5)$ is bounded in the space of observations $\mathcal{Y}$ :

$$
\|\xi\|_{\mathcal{Y}}^{2} \leq \mu^{2}
$$

To formulate the observation problem we need the notion of information or consistency set ([7], [10]).

DeFINITION 1 The information set $U(\tilde{t} ; y(\cdot))$ is the union of all states $\left\{u(\cdot, \tilde{t}), u_{t}(\cdot, \tilde{t})\right\}$ of system (3) at time $\tilde{t}$, for which there exists a tuple $\mathcal{D}=\left\{\Phi(\cdot), \Psi(\cdot), \mu_{1}(\cdot), \mu_{2}(\cdot), f(\cdot, \cdot), \xi(\cdot)\right\} \quad$ (initial conditions, boundary conditions and input and measurement disturbance), satisfying $(4,6)$ (or $(5,6)$ ) and consistent with observation $y(\cdot)$ due to equations $(3,4)$ (or $(3,5))$.

Problem A Find $U(0, y(\cdot))$ - the set-valued estimate, or produce a pointwise estimate for the state $u(0, \cdot)$.

With no bounds given for initial and boundary conditions and no disturbance $f$ the information set $U(0, y(\cdot))$ may turn to be unbounded even with bounded measurement noise. This may happen, for example when the observation is pointwise, at a rational point $x^{0}$. Nevertheless, with bound (6) modified to include all elements of the tuple $\mathcal{D}$, the estimation Problem A makes sense even in the latter case.

A preferable type of solution to Problem A is a recurrent "guaranteed filtering" equation which describes the evolution of the estimate in time, on one hand, and also ensures numerical stability of the corresponding algorithm. A problem closely connected with Problem A is the one of observability of system $(3,4)$ ( or $(3,5)$ ).

Problem B Assume disturbances $f(\cdot, \cdot)=0, \xi(\cdot)=0$. In the absence of information on input - initial conditions $\{\Phi(\cdot), \Psi(\cdot)\}$ and boundary values $\mu_{1}(\cdot), \mu_{2}(\cdot)$ - determine conditions for solvability of the problem: given measurement $y(t), t \in[0, T]$, find output - the solution $u(\cdot, T)$ at time $T$.

The solution to this Problem B gives the so-called "observability condition" for system $(3,4)$ (or $(3,5)$ ) with given type of measurement sensor $G$. It is important to understand which types of sensors ensure observability. 


\section{Some Properties of the Telegraph Equation}

To produce our solutions we need some properties of the telegraph equation

The solution formula

THEOREm 2 (Ladyzhenskaya) Let $\Phi \in H_{0}^{1}, \Psi \in L_{2}(\Omega), f \in L_{2,1}\left(Q_{T}\right)$, $\mu_{1}(t)=\mu_{2}(t) \equiv 0 \Rightarrow$ there exists a unique solution of (3) from $H_{1}\left(Q_{T}\right)$.

Here $H_{1}(\Omega)=\left\{\varphi \mid \varphi, \frac{\partial \varphi}{\partial x} \in L_{2}(\Omega)\right\}$, and $H_{0}^{1}$ is a subspace of $H_{1}(\Omega)$ where smooth functions with compact support form a dense set.

The solution of equation (3) can be written out through Green function, the latter being equal to

$$
G(x, \xi, t)=-\frac{2}{l} \sum_{n=1}^{\infty} \sin \frac{\pi n x}{l} \sin \frac{\pi n \xi}{l} \frac{v_{0}^{2} e^{\frac{\sigma_{1}+\sigma_{2}}{2} t}}{\sqrt{\nu_{n}}} \operatorname{sh} \sqrt{\nu_{n}} t
$$

Here the frequencies $\nu_{n}$ are equal to: $\nu_{n}=\left(\frac{\sigma_{1}-\sigma_{2}}{2}\right)^{2}-\left(v_{0} \frac{\pi n}{l}\right)^{2}$ due to wave dispersion. Using [1], the following theorem can be proved

ThEOREM 3 For $\sigma<\sigma_{\max }(1)$ the system $\left\{e^{i \lambda_{n} t}\right\}, \lambda_{ \pm n}= \pm \sqrt{-\nu_{n}}$ is a Riesz basis in $L_{2}\left(0, T_{0}\right), T_{0}=\frac{2 v_{0}}{l}$;

For other values of $\sigma \exists n_{0}: \sigma_{\max }\left(n_{0}\right) \leq \sigma<\sigma_{\max }\left(n_{0}+1\right)$, in this case the system $\left\{e^{i \lambda_{n} t}\right\}$, where $\lambda_{ \pm n}= \pm \sqrt{-\nu_{n}}, n \geq n_{0}+1 ; \lambda_{ \pm n}= \pm \frac{\pi n v_{0}}{l}, n=$ $0 . . n_{0}$ will be a Riesz basis in $L_{2}\left(0, T_{0}\right)$.

\section{A biorthogonal system}

Let $\sigma<\sigma_{\max }(1)$. We denote by

$$
\varphi_{n}=e^{i \lambda_{n} t}=\cos \lambda_{n} t+i \sin \lambda_{n} t ; \varphi_{-n}=e^{-i \lambda_{n} t}=\cos \lambda_{n} t-i \sin \lambda_{n} t
$$

the system that forms Riesz basis. According to Bari theorem ([1]) there exists a biorthogonal system $\left\{\varphi_{n}^{\prime}\right\}_{n \in Z}$ with uniformly bounded norms, and if $\left\{\varphi_{n}^{0}\right\}_{n \in Z}$ is the orthonormal system from Riesz basis definition (i.e. $\left.\varphi_{n}=V \varphi_{n}^{0}\right)$, then: $\varphi_{n}^{\prime}=\left(V^{-1}\right)^{*} \varphi_{n}^{0} \quad n \in Z$. It is possible to show that orthonormal system can be taken as

$$
\left\{\varphi_{n}^{0}\right\}_{-\infty}^{\infty}=\left\{\cos \frac{v_{0} \pi n}{l} t+i \sin \frac{v_{0} \pi n}{l} t\right\}_{-\infty}^{\infty}
$$

and $V^{-1}$ will be bounded according to the same Bari theorem. In this case biorthogonal system elements $\left\{\varphi_{n}^{\prime}\right\}_{n \in Z}$ can be constructed, and so it is also possible to construct system biorthogonal to $\left\{\sin \lambda_{n} t, \cos \lambda_{n} t\right\}$ :

$$
\begin{array}{ll}
\psi_{n}=\cos \lambda_{n} t=\frac{\varphi_{n}+\varphi_{-n}}{2}, n \in N & \phi_{n}=\sin \lambda_{n} t=\frac{\varphi_{n}-\varphi_{-n}}{2 i}, n \in N \\
\Rightarrow \psi_{n}^{\prime}=\varphi_{n}^{\prime}+\varphi_{-n}^{\prime} & \Rightarrow \phi_{n}^{\prime}=\left(\varphi_{n}^{\prime}-\varphi_{-n}^{\prime}\right) i \Rightarrow \\
<\psi_{n}, \psi_{k}^{\prime}>=T_{0} \delta_{n}^{k}, n, k \in N & <\phi_{n}, \phi_{k}^{\prime}>=T_{0} \delta_{n}^{k}, n, k \in N
\end{array}
$$




\section{Observability}

In this section the system (3) is taken, with $f=\mu_{1}=\mu_{2}=0$, coupled with observation equation (4) or (5) and bound (6). Let us first introduce several definitions (see $[8]$ ).

Definition 4 The system (3),(4) (or (3),(5)) with $f=\mu_{1}=\mu_{2}=0$ is said to be weakly observable if for any signal $y(\cdot)$ observed due to equation (4) (or (5)) with zero disturbance $(\xi=0)$ there exists only one possible couple of initial conditions $\{\Phi, \Psi\}$ that generate the solution $u(\cdot, \cdot)$ which provides the signal $y(\cdot)$.

\section{Distributed observation of state and velocity at time $t_{1}$}

This is the simplest situation. Let $f=0, \mu_{i}=0$ and the observation equations be as follows:

$$
\begin{array}{ll}
y_{1}(\cdot)=u\left(\cdot, t_{1}\right)+\xi_{1}(\cdot) & y_{1}, \quad \xi_{1} \in \mathcal{Y}_{1}=L_{2}(0, l) \\
y_{2}(\cdot)=u_{t}\left(\cdot, t_{1}\right)+\xi_{2}(\cdot) & y_{2}, \quad \xi_{2} \in \mathcal{Y}_{2}=L_{2}(0, l)
\end{array}
$$

Expanding $\Phi, \Psi, y_{i}$ and $\xi_{i}$ into Fourier series over functions $\{\sin (\pi n x / l)\}$, and denoting corresponding Fourier coefficients by $\Phi^{n}$, $\Psi^{n}, y_{n}^{1}, y_{n}^{2}, \xi_{n}^{1}, \xi_{n}^{2}$, we have:

$$
\begin{aligned}
y_{n}^{1} & =\frac{2}{l} \frac{1}{\sqrt{\nu_{n}}} e^{\frac{\sigma_{1}+\sigma_{2}}{2} t_{1}}\left\{\operatorname{sh} \sqrt{\nu_{n}} t_{1} \Psi^{n}+\left(\sqrt{\nu_{n}} \operatorname{ch} \sqrt{\nu_{n}} t_{1}-\right.\right. \\
& \left.\left.-\frac{\sigma_{1} \frac{\sigma_{\sigma}}{2}}{2} \operatorname{sh} \sqrt{\nu_{n}} t_{1}\right) \Phi^{n}\right\}+\xi_{n}^{0} \\
y_{n}^{2} & =\frac{2}{l} \frac{1}{\sqrt{\nu_{n}}} e^{\frac{\sigma_{1}+\sigma_{2}}{2} t_{1}}\left\{\frac{\sigma_{1}+\sigma_{2}}{2} \operatorname{sh} \sqrt{\nu_{n}} t_{1} \Psi^{n}+\sqrt{\nu_{n}} \operatorname{ch} \sqrt{\nu_{n}} t_{1} \Psi^{n}-\right. \\
& \left.\left.-\left(\left(\frac{\nu_{0} \pi n}{l}\right)^{2}+\sigma_{1} \sigma_{2}\right) \operatorname{sh} \sqrt{\nu_{n}} t_{1}\right) \Phi^{n}\right\}+\xi_{n}^{1}
\end{aligned}
$$

The last system is always solvable and the Fourier components of initial state $\Phi, \Psi$ will be

$$
\begin{aligned}
\Phi^{n}= & -\frac{l}{2 \sqrt{\nu_{n}}} e^{-\frac{\sigma_{1}+\sigma_{2}}{2} t_{1}}\left(( y _ { n } ^ { 0 } - \xi _ { n } ^ { 0 } ) \left(-\frac{\sigma_{1}+\sigma_{2}}{2} \operatorname{sh} \sqrt{\nu_{n}} t_{1}-\right.\right. \\
& \left.\left.-\sqrt{\nu_{n}} \operatorname{ch} \sqrt{\nu_{n}} t_{1}\right)+\left(y_{n}^{1}-\xi_{n}^{1}\right) \operatorname{sh} \sqrt{\nu_{n}} t_{1}\right) \\
\Psi^{n}= & -\frac{l}{2 \sqrt{\nu_{n}}} e^{-\frac{\sigma_{1}+\sigma_{2}}{2} t_{1}}\left(( y _ { n } ^ { 1 } - \xi _ { n } ^ { 1 } ) \left(\frac{\sigma_{1}+\sigma_{2}}{2} \operatorname{sh} \sqrt{\nu_{n}} t_{1}-\right.\right. \\
& \left.\left.-\sqrt{\nu_{n}} \operatorname{ch} \sqrt{\nu_{n}} t_{1}\right)-\left(y_{n}^{0}-\xi_{n}^{0}\right)\left(\sigma_{1} \sigma_{2}+\left(\frac{v_{0} \pi n}{l}\right)^{2}\right) \operatorname{sh} \sqrt{\nu_{n}} t_{1}\right)
\end{aligned}
$$

Hence, it follows that

TheOREM 5 For sensor (9) the system is weakly observable.

It is also interesting to investigate the property of strong observability. 
Definition 6 The system (3), (4), (6) (or (3), (5), (6)) with $f=$ $\mu_{1}=\mu_{2}=0$ is said to be strongly observable if the information set of system initial states $U(0, y(\cdot))(p .179)$ is a bounded set in $L_{2}(\Omega)$, whatever be the measurement $y(\cdot)$.

To prove strong observability it is necessary that the series with components (9) converge. For $y_{1}, y_{2} \in L_{2}(0, l)$ the series $\sum_{n=1}^{\infty}\left(\Psi^{n}\right)^{2}$ may not converge because in the expression for $\Psi^{n}$ there is a component $\frac{n^{2}}{\sqrt{\nu_{n}}} \operatorname{sh} \sqrt{\nu_{n}} t_{1} \sim n \sin \sqrt{-\nu_{n}} t_{1}$. In general this series does not converge. But if $t_{1}$ is such that:

$$
t_{1}: \frac{v_{0} t_{1}}{l} \in \mathbf{Z}
$$

where $\mathbf{Z}$ is the set of integers, then due to the properties of the eigenvalues $\nu_{n}$ we have:

$$
\sin \sqrt{-\nu_{n}} t_{1}=-\left(\frac{\sigma_{1}-\sigma_{2}}{2}\right)^{2} \frac{l t_{1}}{2 v_{0} \pi n}+O\left(\frac{1}{n^{3}}\right)
$$

THEOREM 7 For sensor (9) the information domain of initial states $\Phi$ is bounded in $L_{2}(0, l)$. If instant $t_{1}$ satisfies (10), then the information domain for initial values of derivatives $u_{t}(\Psi)$ will also be bounded in $L_{2}(0, l)$. If $(10)$ does not hold, then one can only claim that the last set is bounded in $V^{*}=\left(H^{1}(0, l)\right)^{*}$

Distributed observation of state at two instants of time $t_{1}, t_{2}$ Take $f=\mu_{1}=\mu_{2}=0$ with observation equations as

$$
\begin{array}{ll}
y_{1}(\cdot)=u\left(\cdot, t_{1}\right)+\xi_{1}(\cdot) & y_{1}, \xi_{1} \in \mathcal{Y}_{1}=L_{2}(0, l) \\
y_{2}(\cdot)=u\left(\cdot, t_{2}\right)+\xi_{2}(\cdot) & y_{2}, \xi_{2} \in \mathcal{Y}_{2}=L_{2}(0, l)
\end{array}
$$

Here a system similar to the one in previous section can be written down, but now its discriminant $D=\frac{4}{l^{2} \sqrt{\nu_{n}}} e^{\left(\sigma_{1}+\sigma_{2}\right) t_{1}} \operatorname{sh} \sqrt{\nu_{n}}\left(t_{2}-t_{1}\right)$. For weak observability it is necessary and sufficient that discriminant $D \neq 0$.

$$
t_{2}-t_{1} \notin\left\{\frac{\pi m}{-\sqrt{\nu_{n}}}\right\}_{n, m=1}^{\infty}
$$

TheOREM 8 For sensor (11) system (3) is weakly observable $\Leftrightarrow$ (12).

Spatially averaged observations Let $f=\mu_{1}=\mu_{2}=0$ with observation equation as

$$
y(t)=\int_{0}^{l} u(x, t) w(x) d x+\xi(t) \quad y, \xi \in \mathcal{Y}_{1}=L_{2}\left(0, T_{0}\right)
$$


If now $w_{n}$ are the Fourier coordinates of the weighting function $w(x)$ and $T \geq T_{0}$, multiplying observation equation by function $e^{-\frac{\sigma_{1}+\sigma_{2}}{2} t}$, and calculating scalar products with biorthogonal system functions (8) on $\left[0, T_{0}\right]$, we result in

$$
\begin{aligned}
& y_{n, 1}=\frac{2}{l} w_{n} \cdot\left(\nu_{n}\right)^{-1 / 2} \cdot T_{0}\left\{\Psi^{n}-\frac{\sigma_{1}+\sigma_{2}}{2} \Phi^{n}\right\}+\xi_{n, 1} \\
& y_{n, 2}=\frac{2}{l} w_{n} \cdot\left(\nu_{n}\right)^{-1 / 2} \cdot T_{0}\left\{\sqrt{\nu_{n}} \Phi^{n}\right\}^{+}+\xi_{n, 2}
\end{aligned}
$$

Here $y_{n, i}, \xi_{n, i}$ are scalar products of observations and disturbances with biorthogonal system functions with weight $e^{-\frac{\sigma_{1}+\sigma_{2}}{2} t}$. The initial state can be calculated as:

$$
\begin{aligned}
\Phi^{n} & =\frac{y_{n, 1}-\xi_{n, 1}}{T_{0} w_{n}} \cdot \frac{l}{2} \\
\Psi^{n} & =\frac{\sqrt{\nu_{n}}\left(y_{n, 0}-\xi_{n, 0}\right)-\frac{\sigma_{1}+\sigma_{2}}{2}\left(y_{n, 1}-\xi_{n, 1}\right)}{T_{0} w_{n}} \cdot \frac{l}{2}
\end{aligned}
$$

THEOREM 9 System (3) with sensor (13) having its coefficients $w_{n} \neq 0$ for all $n$ is weakly observable for $T \geq T_{0}$.

DEFINITION 10 The system (3), (4), (6) (or (3), (5), (6)) with $f=$ $\mu_{1}=\mu_{2}=0$ is said to be $\varepsilon$-observable if the projection of the information set $U(0, y(\cdot))$ (p. 179) on any finite-dimensional subspace $X_{r}(0, l)=\operatorname{Span}\left\{w_{n_{j}}(\cdot)\right\}_{j=1}^{r}$ is bounded, whatever be the measurement $y(\cdot)$.

Here system $\left\{w_{n_{j}}\right\}_{j=1}^{r}$ is the set of $r$ arbitrary different functions from the system of eigenfunctions $\{\sqrt{2 / l} \sin (\pi n x / l)\}_{n=1}^{\infty}$.

THEOREM 11 The system with sensor (13) which satisfies $w_{n} \neq 0 n \leq$ $N$ is observable in its first $N$ Fourier components (its first $N$ "harmonics") for $T \geq T_{0}$.

COROLlary 12 If the coefficients $w_{n}$ are non-zero $\forall n$, and $T \geq T_{0}$, then system (3) is observable the in first $N$ Fourier components for any $N$ and therefore $\varepsilon$-observable.

\section{A pointwise sensor at point $x_{0}$}

Here the sensor equation is

$$
y(\cdot)=u\left(x_{0}, \cdot\right)+\xi(\cdot) \quad y, \xi \in \mathcal{Y}_{1}=L_{2}(0, T)
$$

Along the lines of previous procedures, we also come to the next propositions. 
THEOREM 13 System (3) with sensor (14) where $x_{0} / l$ is irrational is weakly observable for $T \geq T_{0}$.

THEOREM 14 System (3) with sensor (14) which satisfies $\cos \frac{\pi n x_{0}}{l} \neq$ $0, n \leq N$, is observable in its first $N$ Fourier components for $T \geq T_{0}$. Moreover, if $x_{0} / l$ is an irrational point, then the system is observable in its first $N$ Fourier components for any $N$, and therefore is $\varepsilon$-observable.

\section{The Filtering Equations}

\section{State Estimation}

Consider the problem of dynamic state estimation for the telegraph equation

$$
\begin{gathered}
u_{x x}=\frac{1}{v_{0}^{2}} u_{t t}-\left(\frac{\sigma_{1}+\sigma_{2}}{v_{0}^{2}}\right) \frac{\partial u}{\partial t}+\frac{\sigma_{1} \sigma_{2}}{v_{0}^{2}} u \\
\left.u\right|_{t=0}=\Phi(x)-? \\
\left.u_{t}\right|_{t=0}=\Psi(x)-? \\
\left.u\right|_{x=0}=\left.0 \quad u\right|_{x=l}=0 \\
\quad y(t)=G(t) u(\cdot, t)+\xi(t) \\
\quad\|\xi\| \leq \mu^{2}
\end{gathered}
$$

The functional describing the measure of uncertainty in the system is taken as:

$$
\begin{aligned}
& F(T)=<\Phi-\Phi^{0}, N_{1}\left(\Phi-\Phi^{0}\right)>+<\Psi-\Psi^{0}, N_{2}\left(\Psi-\Psi^{0}\right)>+ \\
& \quad+<y(\cdot)-G(\cdot) u(\cdot, \cdot), M(\cdot)(y(\cdot)-G(\cdot) u(\cdot, \cdot))>_{L_{2}((0, l) \times(0, T))}
\end{aligned}
$$

Here operators $N_{1}, N_{2}$ may be interpreted as regularizers. Introduce operators $S_{1}(\cdot)$ (Green function), $S_{3}(\cdot)$ :

$$
\begin{gathered}
S_{1}(t)=G(x, \xi, t) ; S_{3}(t)=\frac{\partial G(x, \xi, t)}{\partial t}-\left(\sigma_{1}+\sigma_{2}\right) G(x, \xi, t) \\
\Rightarrow u(\cdot, t)=S_{3}(t) \Phi(\cdot)+S_{1}(t) \Psi(\cdot)
\end{gathered}
$$

then functional (17) can be rewritten as (in the last scalar product dots are omitted):

$$
\begin{aligned}
& F(T)=<\Phi-\Phi^{0}, N_{1}\left(\Phi-\Phi^{0}\right)>+<\Psi-\Psi^{0}, N_{2}\left(\Psi-\Psi^{0}\right)>+ \\
& \quad+<y-G S_{3} \Phi-G S_{1} \Psi, M\left(y-G S_{3} \Phi-G S_{1} \Psi\right)>_{L_{2}((0, l) \times(0, T))}
\end{aligned}
$$

If $u_{0}(T)$ and $u_{1}(T)$ are the minimizers $\Phi, \Psi$ of (19), and if we denote $u(t, T)$ to be the backward solution generated by these minimizers, i.e., 
$u(t, T)=S_{3}(t) u_{0}(T)+S_{1}(t) u_{1}(T)$, then the minimizers should satisfy

$$
\begin{aligned}
& u_{0}(T)=\Phi^{0}+N_{1}^{-1} \int_{0}^{T} S_{3}^{*}(t) G^{*}(t) M(t)[y(t)-G(t) u(t, T)] d t \\
& u_{1}(T)=\Psi^{0}+N_{2}^{-1} \int_{0}^{T} S_{1}^{*}(t) G^{*}(t) M(t)[y(t)-G(t) u(t, T)] d t
\end{aligned}
$$

Now denoting $u(T, T)$ by $\tilde{u}(T)$, differentiating (20) with respect to $T$, we get for $u(t, T)$ :

$$
\begin{aligned}
& \frac{\partial u(t, T)}{\partial T}=\left[S_{3}(t) N_{1}^{-1} S_{3}^{*}(T)+S_{1}(t) N_{2}^{-1} S_{1}^{*}(T)\right] \times \\
& \quad \times G^{*}(T) M(T)[y(T)-G(T) \tilde{u}(T)]- \\
& \quad-\int_{0}^{T}\left[S_{3}(t) N_{1}^{-1} S_{3}^{*}(\tau)+S_{1}(t) N_{2}^{-1} S_{1}^{*}(\tau)\right] G^{*}(\tau) M(\tau) G(\tau) \frac{\partial u(\tau, T)}{\partial T} d \tau
\end{aligned}
$$

If $K(t, T)$ is an operator solving the previous Fredholm equation, then

$$
\frac{\partial u(t, T)}{\partial T}=K(t, T) G^{*}(T) M(T)[y(T)-G(T) \tilde{u}(T)]
$$

and

$$
\begin{aligned}
K(t, T)= & {\left[S_{3}(t) N_{1}^{-1} S_{3}^{*}(T)+S_{1}(t) N_{2}^{-1} S_{1}^{*}(T)\right]-\int_{0}^{T}\left[S_{3}(t) N_{1}^{-1} S_{3}^{*}(\tau)+\right.} \\
& \left.+S_{1}(t) N_{2}^{-1} S_{1}^{*}(\tau)\right] G^{*}(\tau) M(\tau) G(\tau) K(\tau, T) d \tau
\end{aligned}
$$

Using notation $P(T)=K(T, T)$, and denoting

$$
\Xi(T)=G^{*}(T) M(T)(y(T)-G(T) \tilde{u}(T)) \quad \mathcal{D} \cdot=v_{0}^{2} \frac{\partial^{2}}{\partial x^{2}}-\sigma_{1} \sigma_{2}
$$

we finally come to the following system describing the dynamics of the state estimate and the estimates of initial conditions:

$$
\begin{aligned}
& \frac{\partial^{2} \tilde{u}}{\partial T^{2}}-\left(\sigma_{1}+\sigma_{2}\right) \frac{\partial \tilde{u}}{\partial T}=\mathcal{D} \tilde{u}+P(T) \frac{\partial \Xi(T)}{\partial T}+ \\
& +\left[2 \frac{\partial P(T)}{\partial T}-\left.\frac{\partial K(t, T)}{\partial T}\right|_{t=T}-\left(\sigma_{1}+\sigma_{2}\right) P(T)\right] \Xi(T) \\
& \frac{\partial u_{0}}{\partial T}=N_{1}^{-1}\left\{S_{3}^{*}(T)-\int_{0}^{T} S_{3}^{*}(t) G^{*}(t) M(t) G(t) K(t, T) d t\right\} \cdot \Xi(T) \\
& \frac{\partial u_{1}}{\partial T}=N_{2}^{-1}\left\{\begin{array}{c}
\left.S_{1}^{*}(T)-\int_{0}^{T} S_{1}^{*}(t) G^{*}(t) M(t) G(t) K(t, T) d t\right\} \cdot \Xi(T) \\
\tilde{u}(0)=0
\end{array} u_{0}(0)=\Phi^{0} \quad u_{1}(0)=\Psi^{0}\right.
\end{aligned}
$$


The equation for $P(T)$ can also be written as follows

$$
\begin{aligned}
& \frac{\partial^{2} P}{\partial T^{2}}-\left(\sigma_{1}+\sigma_{2}\right) \frac{\partial P}{\partial T}=\mathcal{D} P+P\left[\mathcal{D}^{*}+\left(\sigma_{1}+\sigma_{2}\right) G^{*} M G P-\right. \\
& \left.\quad-\left(G^{*} M G P\right)_{T}^{\prime}-G^{*} M G P G^{*} M G P\right]-\left.\frac{\partial K(t, T)}{\partial T}\right|_{t=T} G^{*} M G P \\
& P(0)=\left.N_{1}^{-1} \quad \frac{\partial P}{\partial T}\right|_{T=0}=N_{1}^{-1} G^{*}(0) M(0) G(0) N_{1}^{-1}
\end{aligned}
$$

And for $K(t, T)$ :

$$
\begin{aligned}
\frac{\partial^{2} K(t, T)}{\partial T^{2}} & -\left(\sigma_{1}+\sigma_{2}\right) \frac{\partial K(t, T)}{\partial T}+\frac{\partial^{2} K(t, T)}{\partial t^{2}}-\left(\sigma_{1}+\sigma_{2}\right) \frac{\partial K(t, T)}{\partial t}= \\
& =\mathcal{D} K(t, T)+K(t, T) \mathcal{D}^{*}+K(t, T)\left[\left(\sigma_{1}+\sigma_{2}\right) G^{*} M G P-\right. \\
& \left.-\left(G^{*} M G P\right)_{T}^{\prime}-G^{*} M G P G^{*} M G P\right]-\frac{\partial K(t, T)}{\partial T} G^{*} M G P
\end{aligned}
$$

The initial conditions for $K(t, T)$ can be obtained from equation (23).

The Dynamic Estimate of Initial Conditions. Consider the problem of dynamic initial state estimation for the telegraph equation. This problem is directly related to the observability property, since for the formulation of the filtering equations the existence of bounded inverse for certain operators is necessary. The related operators are invertible if the property of strong observability is true. These conditions are precisely the ones discussed in the observability sections. Such types of conditions were earlier introduced by J.L.Lions ([4]) and further studied in [3].

Consider problem $(15,16)$. Let the measure of uncertainty in the system be the same as in (6):

$$
\begin{aligned}
F(T) & =\|\xi\|^{2}= \\
& =<y(\cdot)-G(\cdot) u(\cdot, \cdot), M(\cdot)(y(\cdot)-G(\cdot) u(\cdot, \cdot))>_{L_{2}((0, l) \times(0, T))}
\end{aligned}
$$

If we again use the notations $S_{1}(\cdot), S_{3}(\cdot)$ (see (18)), the functional (28) can be rewritten as:

$$
\begin{aligned}
F(T)=<y(\cdot)-G(\cdot) S_{3}(\cdot) \Phi & -G(\cdot) S_{1}(\cdot) \Psi, M(\cdot)(y(\cdot)- \\
& \left.-G(\cdot) S_{3}(\cdot) \Phi-G(\cdot) S_{1}(\cdot) \Psi\right)>
\end{aligned}
$$


If $u_{0}(T)$ and $u_{1}(T)$ are the functions $\Phi, \Psi$ which minimize (29) for each given $T$, they satisfy relations

$$
\begin{aligned}
& \int_{0}^{T} S_{3}^{*}(t) G^{*}(t) M(t)\left[y-G(t) S_{3}(t) u_{0}(T)-G(t) S_{1}(t) u_{1}(T)\right] d t=0 \\
& \int_{0}^{T} S_{1}^{*}(t) G^{*}(t) M(t)\left[y-G(t) S_{3}(t) u_{0}(T)-G(t) S_{1}(t) u_{1}(T)\right] d t=0
\end{aligned}
$$

If the system (15-16) is strongly observable, then expressing the minimizers from (30) and differentiating them with respect to $T$ it is possible to write down the following system of evolution equations:

$$
\begin{aligned}
& \frac{\partial u_{0}}{\partial T}=K_{3}(T) u_{0}(T)+\mathcal{K}_{3}(T)\left\{y(T)-G(T) S_{1}(T) u_{1}(T)\right\}-\mathcal{M}_{3}(T) \frac{\partial u_{1}(T)}{\partial T} \\
& \frac{\partial u_{1}}{\partial T}=K_{1}(T) u_{1}(T)+\mathcal{K}_{1}(T)\left\{y(T)-G(T) S_{3}(T) u_{0}(T)\right\}-\mathcal{M}_{1}(T) \frac{\partial u_{0}(T)}{\partial T} \\
& u_{0}(0)=0 \quad u_{1}(0)=0
\end{aligned}
$$

Here $K_{i}(t)=S_{i}^{*}(t) G^{*}(t) M(t) G(t) S_{i}(t), \mathcal{K}_{i}(T)=\left(\int_{0}^{T} K_{i}(t) d t\right)^{-1} S_{i}^{*}(T) \times$ $\times G(T) M(T), \mathcal{M}_{i}(T)=\left(\int_{0}^{T} K_{i}(t) d t\right)^{-1} \int_{0}^{T} S_{i}^{*}(t) G^{*}(t) M(t) G(t) S_{j}(t) d t$ where $i, j=1,3$ and $i \neq j$.

If system $(15,16)$ is only $\varepsilon$-observable, then the projections of the informational domain on any finite-dimensional subspace are bounded. It is therefore possible to indicate the same types of filtering equations in finite-dimensional space. These would be written in terms of respective Fourier coefficients.

\section{The Duality of Optimal Control and Observation problems}

Consider the observability problem for the telegraph equation:

$$
\begin{aligned}
& \frac{\partial^{2} u}{\partial t^{2}}-\left(\sigma_{1}+\sigma_{2}\right) \frac{\partial u}{\partial t}=\mathcal{D} u \\
& \left.u\right|_{\partial \Omega}=\left.0 \quad u\right|_{t=0}=\left.\Phi \quad u_{t}\right|_{t=0}=\Psi \\
& y=G u+\xi \\
& \|\xi\| \leq \mu
\end{aligned}
$$

Here operator $\mathcal{D}$ is defined by (24). If the solution is presented as (18), the support function of the information set $U$ is as follows:

$$
\begin{aligned}
& \rho(l, U(0, T))=\sup \left(<\Phi, S_{3}^{*}(T) l>+<\Psi, S_{1}^{*}(T) l>+<\psi(\cdot), y(\cdot)-\right. \\
& \left.-G(\cdot) S_{1}(\cdot) \Psi-G(\cdot) S_{3}(\cdot) \Phi-\xi(\cdot)>_{L_{2}(Y \times[0, T])} \mid\|G u-y\| \leq \mu\right)=
\end{aligned}
$$

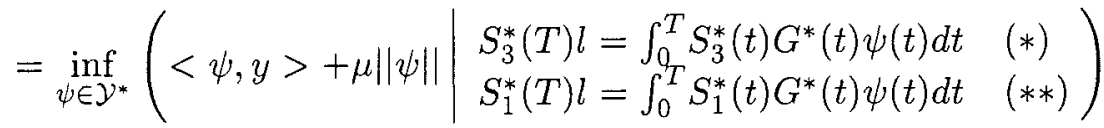


Since for the telegraph equation the Green function is $S_{1}(t, \cdot)$, equation $\left({ }^{*}\right)$ is the solution to the adjoint equation in inverse time with right-hand side $G^{*} \psi$ :

$$
\begin{aligned}
& \frac{\partial^{2} v}{\partial t^{2}}+\left(\sigma_{1}+\sigma_{2}\right) \frac{\partial v}{\partial t}=\mathcal{D} v+G^{*}(t) \psi(t, \cdot) \\
& \left.v\right|_{\partial \Omega}=\left.0 \quad v\right|_{t=T}=\left.0 \quad v_{t}\right|_{t=T}=0 \\
& \left.v\right|_{t=0}=S_{1}^{*}(T, \cdot) l(\cdot) .
\end{aligned}
$$

This leads to a control problem for system (32), where $\psi(t, \cdot) \in \mathcal{Y}^{*}$ is the control. Due to $S_{1}(0)=0$ the controllability of the following system will be equivalent to the solvability of equation $\left({ }^{* *}\right)$ :

$$
\begin{aligned}
& \frac{\partial^{2} v}{\partial t^{2}} F+\left(\sigma_{1}+\sigma_{2}\right) \frac{\partial v}{\partial t}=\mathcal{D} v-\frac{\partial}{\partial t}\left\{G^{*}(t) \psi(t, \cdot)\right\}-\left(\sigma_{1}+\sigma_{2}\right) G^{*}(t) \psi(t, \cdot) \\
& \left.v\right|_{\partial \Omega}=\left.0 \quad v\right|_{t=T}=\left.0 \quad v_{t}\right|_{t=T}=0 \\
& \left.v\right|_{t=0}=S_{3}^{*}(T, \cdot) l(\cdot)-S_{1}^{*}(T) G^{*}(T) \psi(T), \\
& \text { where } \psi(t, \cdot) \in \mathcal{Y}^{*} \text { is the control. }
\end{aligned}
$$

This is a control problem for system (33) in backward time.

Thus, the solution of the observation problem for system (31) under disturbances (noise) is equivalent to finding the control $\psi(t, \cdot) \in \mathcal{Y}^{*}$, which simultaneously steers the adjoint systems (32), (33) in backward time to the prescribed end-points, as given in (32), (33), under minimum of the norm conjugate to the one that bounds the observation noise ( [4]). The prerequisite of such properties for finite-dimensional systems was given in [5], [6] and for infinite-dimensional time lag systems in [9].

\section{References}

[1] S.A. Avdonin and S.A. Ivanov. Families of exponentials: the method of moments in controllability problems for distributed parameter systems. Cambridge University Press, 1995.

[2] R. Feynmann, R.B. Leighton, and M. Sands. The Feynmann Lectures in Physics. Addison-Wesley, Reading, 1963.

[3] L.H. Ho. Observabilité frontière des equations des ondes. Comptes Rendus de lÁcademie des Sciences de Paris, 302:443-446, 1986.

[4] Lions J-L. Contrôlabilité exacte, perturbations et stabilisation de systèmes distribués, volume Tome 1 Controlabilité Exacte. Masson, 1988.

[5] R.E. Kalman. On the general theory of control systems, 1960. Proc. of the 1-st IFAC CONGRESS, v.1, Butterworths, London. 
[6] N.N. Krasovski. The theory of control of motion (in Russian). M.: Nauka, 1968.

[7] A.B. Kurzhanski and A.Yu. Khapalov. An observation theory for distributedparameter systems. Journal of Math.Systems, Estimation and Control, 1, 1990.

[8] A.B. Kurzhanski and I.F. Sivergina. $\epsilon$-observability of distributed parameter systems, 1992. Trudy IMM UrO RAN, v. 1, pp. 122-137.

[9] A.B. Kurzhansky. On duality of problems of optimal control and observation. J. Appl. Math Mechs, 34, No. 3:..., 1970.

[10] A.B. Kurzhansky and I.F. Sivergina. On noninvertible evolutionary systems: guaranteed estimates and the regularization problem. Sov.Math.Doklady, 42, No. 2:451-455, 1991. 